\title{
A Comparative Analysis of the Cost Effectiveness of Five Advanced Skin Substitutes in the Treatment of Foot Ulcers in Patients with Diabetes
}

\author{
Robert Snyder ${ }^{1 *}$ and Karim Ead J ${ }^{2}$ \\ ${ }^{1}$ Barry University, 7301 N University Drive, Tamarac, Florida 33321 \\ ${ }^{2}$ Westside Reginal Medical Center, 8201 W Broward Blvd, Plantation, Florida
}

Submission: September 24, 2020; Published: October 01, 2020

"Corresponding author: Robert Snyder, Barry University, 7301 N University Drive, Tamarac, Florida 33321

Abstract

Objective: The purpose of this economic analysis was to generate the cost-effectiveness of five (5) advanced skin substitutes/ Cell/Tissue based Products (CTPs) in the treatment of foot ulcers in patients with diabetes (DFUs): ActiGraft ${ }^{\circ}$ (RedDress, Pardes Hanna, Israel); Apligraf ${ }^{\circ}$ (Organogenesis, Canton, MA); Dermagraft ${ }^{\circledR}$ (Organogenesis, Cant-on, MA) ; Grafix Core $₫($ Smith \& Nephew, Andover, MA) and EpiFix $\AA$ (MiMedx, Marietta, GA).

Methods: For each skin substitute product, three data points were central to the analysis: Number of applications over a 12-week course of treatment; complete healing efficacy over a 12-week course of treatment; product cost per application; the number of applications and complete healing efficacy were obtained from seven established published clinical studies. Inclusion/Exclusion criteria included either a randomized controlled trial, prospective cohort, or retrospective cohort design that included at least two study arms; reported wound healing at 12 or 16 weeks, reported number of application of products used to achieve healing and a minimum 40 subjects in the primary treatment arm. Due to the complexities of cost-benefit analysis and difficulties encountered when comparing research (i.e. different run-in periods, numbers of subjects and variable endpoints) the study selection for Apligraf, Dermagraft, Grafix and Epifix used inclusion/exclusion criteria from Samsell et al. For Actigraft, study selection was limited to a pilot study.

Results: A 12-week product cost of the CPT's analyzed ranged from $\$ 2,120$ to $\$ 7,647$. ActiGraft had a product cost advantage ranging from $\$ 1,245$ to $\$ 5,527$ over 12 weeks. The cost premium for other skin substitutes ranged from 59 to $261 \%$. The 4 -week product cost ranged from $\$ 1,060$ to $\$ 4,840$. ActiGraft had a product cost advantage ranging from $\$ 1,710$ to $\$ 3,780$ over 4 weeks. The cost premium for other skin substitutes ranges from 161 to $357 \%$. The weighted average treatment cost ranged from $\$ 5,505$ to $\$ 11,783$. ActiGraft had a cost advantage ranging from $\$ 2,504$ to $\$ 6,278$. The cost premium for other skin substitutes ranges from 45 to $114 \%$. ActiGraft had a cost advantage ranging from $\$ 81$ to $\$ 6,279$ in the hospital outpatient setting and from $\$ 1,245$ to $\$ 5,527$ in the physician office setting. The cost premium for other skin substitutes ranges from 2 to $154 \%$ in the hospital outpatient setting and from 55 to $243 \%$ in the physician office setting. Standardized Effective Analysis (SEA) ranged from 4 to 18 patients per 100 healed in the hospital outpatient setting, and 5 to 32 patients per 100 healed in the physician office setting. ActiGraft had the greatest SEA in both settings. Additionally, ActiGraft offered the lowest cost per $\mathrm{cm} 2$ while delivering the highest healing efficacy. ActiGraft is autologous, prepared from the patient's own whole blood at the point of care. Unlike other CTP's in this analysis ActiGraft had no biologic material wastage.

Conclusion: This head-to-head comparison showed that ActiGraft was more cost efficient as an advanced therapy for DFU's when compared to the four (4) other advanced products analyzed. However, the ActiGraft data sourced from a pilot study presents limitations for comparison with studies meeting Samsell's criteria. It is the authors' intention to update this analysis when future published studies meet this benchmark.

Keyword: Diabetic foot ulcers; Economic analysis; Cell/tissue-based products; comparative analysis

Abbreviations: CTP'S: Cell/Tissue based Products; SEA: Standardized effective analysis; LEA: Lower extremity amputation; CMS: Centers for Medicare and Medicaid Service; PQRS: Physician Quality Reporting System; ITT: Intent-to-treat ; PAR: Percent area reduction; PP: Per-protocol; DFU: diabetic foot ulcers; SOC: standard of care; BLCC: Bioengineered living cellular construct; WAC: Wholesale Acquisition Cost; OPPS: Outpatient Prospective Payment System; WAVG: Weighted average treatment cost

\section{Introduction}

In the United States, it is reported that chronic wounds affect approximately 6.5 million patients. The incidence of chronic wounds is expected to increase as our population ages. The impact of chronic wounds on the health and quality of life of patients and their families should not be underestimated. Wound healing and tissue regeneration represent a well-orchestrated series of events that consists of highly integrated biological and molecular 
processes that includes cellular migration and proliferation, and of extracellular matrix deposition and remodeling. Certain metabolic and pathophysiologic conditions such as foot ulcers in patients with diabetes can deleteriously impact the normal physiologic process so that healing is impaired or delayed, resulting in chronic, non-healing wounds. The financial burden imposed by chronic wounds on the healthcare system cannot be understated. Approximately 15\% of Medicare subpopulations ( 8.2 million) had at least one type of wound or infection. Total Medicare spending estimates for all wound types ranged from $\$ 28.1$ to $\$ 96.8$ billion. Such profound economic trends emphasize the importance of using advanced wound-healing modalities as adjuvant therapies that work synergistically with standard wound care regimens. Advanced treatment modalities that promote rapid and complete healing may reduce the risk of amputation and can substantially improve quality of life while decreasing financial burdens to the individual and society overall. Based upon the paucity of data in this area, the results of a comparative cost analysis could be useful in guiding clinicians towards therapies that are both effective and cost efficient when considering a treatment plan for patients with non-healing lower extremity wounds particularly recalcitrant foot ulcers in patients with diabetes.

There are approximately 34.2 million patients with diabetes in the United States representing $10.5 \%$ of the population. With rates of obesity soaring, it is likely that this number will increase exponentially. In 2017, the total estimated cost of diagnosed diabetes in the US was $\$ 327$ billion [1]. Geiss et al. [2] opined that after years of decline, the rate of amputations increased by 50\% between 2009 and 2015 to 4.6 for every 1000 adults with the highest numbers observed among young adults (age 18-44 years) and those in middle age (45-64 years) . These groups were more likely to be uninsured and disparate populations were 4 times more likely to experience diabetes-related amputation [2]. A systematic review suggested the incidence of lower extremity amputation (LEA) in the diabetic population ranged from 78-704 per 100,000 person years and the relative risks between diabetic and non-diabetic patients varied between 7.4 and 41.3. Therefore, published data are conflicting. However, it is likely that more than $60 \%$ of non-traumatic lower limb amputations occur in patient with diabetes [3].

Furthermore, it remains clear that LEA in this demographic results in higher mortality, reduced quality of life, and increased medical costs. For example, in 2010, 73,000 non-traumatic lower limb amputations were performed in the US due to diabetes, at an average cost of $\$ 70,434$. Brownrigg et al. [4] suggests that a foot ulcer is the initial event in more than $85 \%$ of major amputations performed on people with diabetes. Even more distressing is the possibility that up to $50 \%$ of patients with diabetes who undergo amputation will die within 2 years 7 . Therefore, aggressive and specialized care remains critical when patients with diabetes present with a foot ulcer and must be facilitated in a cost-efficient manner. Skin substitutes/ CTP's are frequently used to treat chronic diabetic foot ulcers and there are numerous advanced skin substitutes on the market. Samsell [5] conducted a retrospective cost analysis comparing eight skin substitutes using previously published efficacy rates combined with the Centers for Medicare and Medicaid Services (CMS) 2018 cost data. They included seven studies that described the overall value rates for treatment of DFU's using the skin substitutes. These studies are actively being used by regulatory bodies, healthcare institutions and reimbursement agencies to generate usage decisions. Due to the complexities of cost-benefit analysis and difficulties encountered when comparing research (i.e. different run-in periods, numbers of subjects and variable endpoints) the study selection for Apligraf, Dermagraft, Grafix and Epifix used inclusion/exclusion criteria from this study.39 However, for Actigraft the study selection was limited to a pilot study.

\section{The Problem}

A seminal study entitled "An Economic Evaluation of the Impact, Cost, and Medicare Policy Implications of Chronic Nonhealing Wounds," analyzed the Medicare 5\% Limited Data Set for calendar year 2014 and determined that chronic nonhealing wounds impact nearly $15 \%$ ( 8.2 million) of Medicare beneficiaries, far more than suggested by previous studies. Furthermore, conservative estimates for total Medicare annual spending for all wound types ranged from $\$ 28.1$ billion to $\$ 31.7$ billion [8]. Treatment and management of infected or re-opened (dehisced) surgical wounds account for the highest per-wound costs. Hospital outpatient care drove the highest site-of-service costs, demonstrating the shift from hospital inpatient to outpatient services in the wound care space. In regard to site of service, hospital outpatient settings drove the greatest proportion of costs - demonstrating a major shift in costs from hospital inpatient to outpatient setting. However, Carter opined that unlike evidence-based medicine, there is less agreement on how to conduct economic analysis in healthcare, and universal guidelines for reporting these studies is lacking and "real-world data is almost non-existent. This has led to a paucity of economic data to support claims of cost benefit that require several key points in decision making (Figure 1).

During the early 2000s, the Centers for Medicare and Medicaid Services (CMS) established a hospital-based outpatient payment system with the purpose of providing continued care most notably in complex cases where patients did not necessarily require hospitalization. However, the established reimbursement system rewarded healthcare providers according to the sheer volume of interventions/procedures performed without a feedback system for quality of care, outcome, or patient-centered concerns. In order to create a financial/reimbursement paradigm shift, in 2006 CMS established the Physician Quality Reporting System (PQRS). With the Medicare patient population, this system integrated incentive payments to qualified healthcare professionals that properly documented quality measure data. However, in 2008 they also 
mandated that any US healthcare provider who fails to successfully report PQRS measures in 2016 will lose $4 \%$ of their Medicare revenue in 2018. CMS also monitors the hospital readmission rates of patients with chronic non-healing wounds [6]. It should be noted that approximately $\$ 17 \mathrm{~B}$ or $65 \%$ of total readmissions are considered avoidable. Consequently, a new round of Medicare readmission penalties impacted $83 \%$ of hospitals thus far in 202013. Furthermore, it is estimated that $1.7 \mathrm{M}$ patients acquire healthcare associated infections. In January 2020, 786 hospitals had their Medicare reimbursement lowered 1\% for having high hospital acquired infection rates. Within the last 10-20 years, the US healthcare system has been experiencing a paradigm shift into a quality-based-system. By 2024, wound care products alone could range from \$15B-\$22B15. In 2006, CMS established the Physician Quality Reporting System (PQRS), which initially provided incentive payments to qualified healthcare professionals reporting quality measure data on Medicare patients [6]. Now moving into the penalty phase, US healthcare providers who fail to successfully report PQRS measures in 2016 will lose 4\% of their Medicare revenue in 2018 [6]. Hospital and nursing care represent the most expensive interventions: Advanced therapies could decrease or obviate the need for hospitalizations or frequent dressing changes.

Key Points for Decision Makers

Few good economic studies exist quantifying the
cost effectiveness of strategic or guideline-based
interventions in regard to chronic wound
management.

The strongest evidence is for prevention and treatment of diabetic foot ulcers, prevention of pressure ulcers in long-term care settings, and treatment of patients with critical limb ischemia.

Clinical practice guidelines are proliferating in the field of chronic wound care, but large gaps exist in demonstrating their cost effectiveness.

Figure 1: Key Points for Decision Makers [32].

\section{Is Standard Care Effective?}

In 1999, Margolis [7] meta-analysis determined the percentage of individuals with neuropathic diabetic foot ulcers receiving good wound care who heal within a defined period of time. After 20 weeks of good wound care, approximately $31 \%$ of diabetic neuropathic ulcers heal [8]. Similarly, after 12 weeks of good care, approximately $24 \%$ of neuropathic ulcers attain complete healing [8]. A recent meta-analysis by Parks et al. suggests that the percentage of diabetic foot ulcers healed with standard of care therapies alone have improved over the past 20 years. Their literature search yielded 16 randomized clinical trials that fit the selection criteria to be included in the meta-analysis [9]. Analysis of the percentage of wounds healed with standard of care therapy alone yielded the following results: 6 weeks $(14.9 \% \pm 13.0 \%)$, 12 weeks $(33.4 \% \pm 18.2 \%), 20$ weeks $(43 \% \pm 0 \%) 17$. Although there has been improvement in healing rates utilizing standard of care, many ulcers in patients with diabetes may require advanced therapies to facilitate closure [9]. Interestingly, a paradigm shift in how advanced therapies are paid appears counter-intuitive to quality measures and pay for performance models (high costlow cost rather than evidence-based). In large part this is due to lack of evidence as it relates to cost effectiveness. Therefore, evidence must be tempered by the small number of available studies including variability in trial duration, baseline wound characteristics, and limitations in study quality. At the end of the day payers need more high-quality head-to-head comparisions to guide coverage and reimbursement allocation.

Due to the systemic implications of chronic non-healing wounds, it is critical that skilled medical personnel incorporate a holistic approach in their treatment pathways. It is important to be cognizant that chronic non-healing wounds are a symptom of a greater systemic disease process. Additionally, the treatment pathway of chronic wounds particularly in patients with diabetes, often involves extended hospital stays and long-term outpatient follow-up visits with costly advanced therapeutic intervention. To date, the strongest evidence is for prevention and treatment of diabetic foot ulcers, prevention of pressure ulcers in long term care settings and treatment of patients with critical limb ischemia. Clinical practice guidelines are proliferating in the field of chronic wound care, but large gaps exist in demonstrating their cost effectiveness. However, the immense economic and social impact of wounds in our society calls for allocation of a higher level of 
attention and resources to understand biological mechanisms underlying cutaneous wound complications.

\section{Cell and Tissue Based Therapies}

When wounds fail to achieve sufficient healing after 4 weeks of standard care, reassessment of underlying pathology and consideration of the need for advanced therapeutic agents should be undertaken. Snyder et al. demonstrated that a $<50 \%$ PAR after 4 weeks of treatment predicts DFU failure to heal by 12 weeks [6]. Therefore, frequent reevaluation of the wound and its treatment is recommended. Advances in cell biology and tissue engineering have led to an increase in the quantity and quality of biological wound dressings. Many cell-based dressings utilize scaffolds which are designed to easily integrate with host tissue and provide an optimal environment for cell growth and differentiation. Cell and tissue-based products (CTP) are among some of the latest biotechnology treatment modalities in medicine. These treatment modalities can be defined as therapy in which cellular material is injected or otherwise transplanted into or on an area of medical concern (i.e. chronic non-healing wounds). CTPs may contain viable or nonviable cells, tissues, proteins, and other materials for which there is a rationale for benefit beyond that achievable with conventional wound coverings. There are three essential categories within the framework of CTP: allogenic, autologous and xenogeneic methodologies.

In autologous based cell therapies, the tissues/cells that are transplanted are derived from the patient's own body. Autologous therapies have gained in popularity especially involving the treatment of foot ulcers in patients with diabetes (DFU's). Some of the key benefits include biocompatibility, reduced risks associated with disease transmission observed with other grafts and cell allografts, and the minimal chance of rejection. For example, ActiGraft TM (RedDress, Israel) is a regenerative wound care solution comprising in vitro blood clots from a patient's whole blood. Once applied, the blood clot tissue serves as a protective covering, biologic scaffold and wound microenvironment to promote the natural wound healing processes of the body [10]. In contrast to other CTP methodologies, autologous blood represents an unlimited resource which can be prepared and utilized immediately as a point of care therapy. Autologous blood tissue possesses several key characteristics of an ideal wound dressing. These include a robust structural framework, a conduit for water vapor transportation, elasticity, creation of bacterial barrier, absence of toxicity and antigenicity, antisepsis, hemostatic activity, ease of application and removal, minimal storage requirements, and reasonable expense.

Snyder [11] studied the safety and efficacy of an autologous blood clot product in the management of Texas $1 \mathrm{~A}$ or $2 \mathrm{~A}$ neuropathic diabetic foot ulcers in a prospective, multicenter, open label pilot study. Twenty patients were enrolled; 20 were analyzed in the intent-to-treat (ITT) population and 18 in the per-protocol (PP) group. The proportion of wounds healed in the ITT and PP populations were 13 out of 20 (65\%) and 13 out of
$18(72.2 \%)$, respectively. Percent area reduction (PAR) for the ITT population at 4 and 12 weeks was $61.6 \%$ and $67.1 \%$, respectively; the PARs for the PP population were $60.3 \%$ and $76.2 \%$ at 4 and 12 weeks, respectively. Mean times to wound healing were 59 days and 56 days in the ITT and PP populations, respectively. This study demonstrated that the blood clot tissue product was safe and efficacious for treating DFUs. An allograft is typically derived from organs, tissues or cells that are donated and developed from a genetically distinct individual of the same species. Some of the more commonly used allogenic CTP's are Apligraf TM (Organogenesis, Canton, MA), Dermagraft TM (Organogenesis, Canton, MA) and homologous use therapies such as the Epifix TM (MiMedix, Marietta, Georgia) derived from amnion and chorion and Grafix Core (Smith \& Nephew, Andover, MA).

\section{Living CTP's}

Apligraf TM is a living bi-layered skin substitute. It is a living active dermal layer that is enriched with human keratinocytes and fibroblasts. This skin substitute is derived from human foreskin in a bovine Type I collagen matrix and a living active epidermal layer formed by human keratinocytes within the stratum corneum [12]. The upper epidermal layer is formed by human keratinocytes (epidermal cells). This advanced product consists of an array of growth factors, cytokines and various proteins essential for wound healing [12]. Falanga et al. [13] conducted the first randomized control study demonstrating Apligraf as an effective and safe mode of treatment for chronic, nonhealing VLU. This multicenter study was completed on 293 patients in an outpatient setting and examined VLU receiving either compression therapy alone compared with compression therapy and serial (up to 5) applications of the human skin equivalent, Apligraf [14]. This study found that treatment with bioengineered skin was more effective than compression therapy at its primary endpoint of 6 months, with $63 \%$ versus $49 \%$ of patients having completely healed, respectively, and the median time to complete wound closure was 61 versus 181 days, respectively, in the two groups [14]. In 2009, Edmonds [12] compared the efficacy and safety of Apligraf in combination with standard therapy versus standard therapy alone in the treatment of neuropathic diabetic foot ulcers. There were 82 randomized treatment groups, and 72 treated (33 Apligraf subjects and 39 standard therapy subjects [15]. By 12 weeks, 51.5\% (17/33) Apligraf subjects had achieved complete wound closure compared with $26.3 \%$ (10/38) of standard therapy subjects [15].

Dermagraft is a living, single layer of human fibroblastderived dermal substitute approved as an adjunctive treatment for recalcitrant foot ulcers in patients with diabetes. Marston [14] performed a randomized, controlled, multicenter study at 35 sites across the United States. Three-hundred and fourteen patients were enrolled. Results demonstrated that foot ulcers in patients with diabetes that were present for greater than 6 weeks showed experienced greater clinical benefit with Dermagraft than patient treated with conventional therapy. At 12 weeks 30.0\% (39 of 130) 
of the Dermagraft patients healed compared to $18.3 \%$ (21 of 115) in the control group ( $\mathrm{p}=0.023)$.

\section{Dehydrated Human Amnion/Chorion Membrane}

Epifix TM is a dehydrated human amnion/chorion membrane (dHACM) allograft. This therapy represents a bioactive tissue matrix allograft composed of dHACM that preserves and contains multiple ECM proteins, growth factors, cytokines, and other specialty proteins27,28. Possible benefits of these cell-based therapies include modulation of chronic inflammatory states, paracrine signaling that stimulates and attracts healthy cells to the wound bed, and the integration of robust effector cells that help to orchestrate wound closure. In some of his earlier research, Zelen [1] facilitated a study to evaluate dehydrated human amniotic membrane (dHAM) allografts in patients with diabetic foot ulcers (DFU). The study population was identified from patients with chronic DFUs enrolled in a randomized trial comparing standard care with standard care with the addition of dHAM. "The study population was identified from patients with chronic DFUs enrolled in a randomized trial comparing standard care with standard care with the addition of dHAM. The present study included patients that failed to heal (defined as $<50 \%$ decrease in wound size after 6 weeks, or not completely healed by 12 weeks of treatment) with standard care. One week after withdrawal from the randomized trial, these patients were offered treatment with standard care and bi-weekly application of dHAM. Each patient was used as their own control to compare wound size reduction between treatment periods, and healing rates within the 12-week dHAM treatment period. Eleven patients were included in the study. Mean wound chronicity was $21.1 \pm$ 12.4 weeks (range 11-54 weeks) and mean wound size was 4.7 $\pm 5.0 \mathrm{~cm}^{2}$, at baseline. Complete healing was achieved in $55 \%$ by 4 weeks, $64 \%$ by 6 weeks and $91 \%$ by 12 weeks with bi-weekly dHAM application. Mean weeks to complete healing was $4.2 \pm 3.1$ weeks for the 10 patients healed. After 4 weeks of standard care, wounds had decreased in size an average of $26.8 \pm 45.3 \%$ versus $87.6 \pm 16.0 \%$ after 4 weeks of dHAM treatment (two applications; $\mathrm{p}<0.001$ ). This study provided evidence that the incorporation of dHAM into standard of care for patients with chronic DFUs can be beneficial and that dHAM is a viable option for the treatment of recalcitrant DFUs".

\section{Cryopreserved Placental Membrane}

Grafix Core (Smith \& Nephew, Andover, MA) is a viable cryopreserved placental membrane (vCPM) used as an adjunctive treatment for recalcitrant foot ulcers. In patients with diabetes (DFU). A multicenter randomized controlled trial revealed that $\mathrm{VCPM}$ resulted in a higher proportion of wound closures when compared to good wound care: $62 \%$ versus $21 \%$ $(\mathrm{p}<0.01)$. Effectiveness was also gleaned from real world data in a retrospective analysis. These results mirrored previous RCT efficacy, thus supporting the benefits of vCPM for DFU management.

\section{What Clinicians and Payers Want?}

Despite evidence based upon prospective analysis, studies rarely address economic efficiency. Clinicians and payers continue to require head to head comparisons when making clinical and economic decisions. Zelen [1] studied a cohort of 100 patients and assessed the efficacy of EpiFix and Apligraf (MiMedx, Marietta, GA); Organogenesis Inc., Canton, MA, USA) versus standard of care (SOC) over a duration of 12 weeks. Inclusion criteria were infection-free ulcers unresponsive to standard wound care for 4 weeks or longer, with adequate circulation to the affected extremity [16]. Complete wound closure was achieved by $97 \%$ of EpiFix patients by week 12, compared to $73 \%$ of subjects treated with Apligraf, and 51\% of subjects receiving SOC alone [16]. Additionally, patients in the EpiFix group used $94.4 \%$ less graft material and an average of 58\% fewer grafts than Apligraf patients, resulting in an $83 \%$ lower median cost of graft material for the EpiFix group compared to the Apligraf group [16]. In 2015, Kirsner conducted the largest comparative effectiveness study examining outcomes of bioengineered living cellular construct (BLCC) and a dehydrated human amnion/chorion membrane allograft (dHACM) for the treatment of DFU's [17]. Using a wound care specific electronic medical record database, real world outcomes were assessed in 218 patients with 226 DFU receiving treatment in 2014 at 99 wound care centers. They found DFUs treated with BLCC had higher rates of healing in less time compared to those treated with dHACM, increasing the probability of healing by $97 \%$ when compared with dHACM $(\mathrm{p}=0.01)$ [17].

There are many studies showing efficacy of advanced products and therapies however these types of studies are often difficult to compare relative to methodologies and run-in periods; many are underpowered. Cost analysis remains either absent or inconsistent. Although head-to-head comparisons should be an essential part of the decision-making process they are rarely undertaken. Identifying the costs associated with treating wounds is rarely simple, as wounds can be related to secondary diagnosis and in vastly different methodologies, end points and the number of subjects. However, as reimbursement moves from a single payer to a value-based model clinicians and payers must have the opportunity to determine whether the higher upfront costs of these therapies are associated with decreased overall cost to the health care system (Figure 2).

Results: Economic Analysis

\section{Overview}

The following economic analysis compares the cost and efficacy of five skin substitutes.

i. $\quad$ ActiGraft ${ }^{\circledR}$ (RedDress, Pardes Hanna, Israel)

ii. Apligraf $₫($ Organogenesis, Canton, MA)

iii. Dermagraft ${ }^{\circledR}($ Organogenesis, Canton, MA)

iv. Grafix Core $\AA$ (Smith \& Nephew, Andover, MA)

v. $\quad$ EpiFix $®(M i M e d x$, Marietta, GA) 


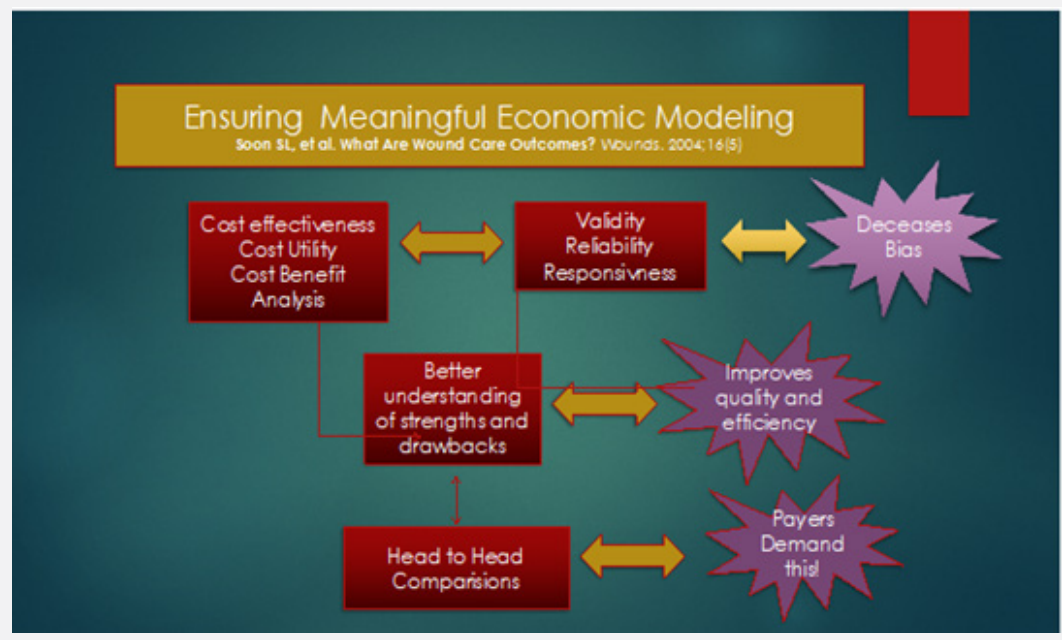

Figure 2: Ensuring Meaningful Economic Modeling.

The analysis is comprised of a series of cost comparisons to treat one diabetic foot ulcer (DFU).

a. 12-Week Product Cost

b. 4-Week Product Cost

c. Weighted Average Treatment Cost for Healed and Unhealed Populations

d. Medicare Reimbursement Cost - Hospital Outpatient and Physician Office Settings

e. Standardized Effectiveness Analysis

f. Scatter Plot - 12-Week Product Cost versus Complete Healing Efficacy

g. Scatter Plot - Cost per Square Centimeter versus Complete Healing Efficacy

\section{Methodology}

For each product, three data points are central to the analysis:

a) Number of applications over a 12-week course of treatment.

b) Complete healing efficacy over a 12 -week course of treatment.

c) Product cost per application.

The number of applications and complete healing efficacy were obtained from the following published clinical studies. Where two studies are listed, data points were derived using a weighted average of the two data points (Table 1). For ActiGraft, study selection was limited to a pilot study. The study selection for Apligraf, Dermagraft, Grafix and EpiFix used inclusion/exclusion criteria defined by Samsell [5] including these key characteristics [5].

Table 1: The number of applications and complete healing efficacy were obtained from the following published clinical studies. Where two studies are listed, data points were derived using a weighted average of the two data points.

\begin{tabular}{|c|c|c|c|}
\hline Product & Study Design & Study & Subjects in Arm (n) \\
\hline ActiGraft & Pilot & Snyder, 2018[34] & 18 \\
\hline Apligraf & Retrospective & Kirsner, 2015[35] & 163 \\
\hline \multirow{2}{*}{ Dermagraft } & RCT & Marston, 2003[36] & 130 \\
\hline Grafix Core & Retrospective & Kraus, 2017[37] & 59 \\
\hline \multirow{2}{*}{ EpiFix } & RCT & Kirsner, 2015[35] & 50 \\
\hline
\end{tabular}

i. Either a randomized controlled trial, prospective cohort, or retrospective cohort design that included at least two study arms.

ii. Reported wound healing at 12 or 16 weeks. iii. Reported number of applications of product used to achieve healing.

iv. Minimum 40 subjects in the primary treatment arm. 
The ActiGraft data sourced from a pilot study presents limitations for comparison with studies meeting Samsell's criteria. It is the authors' intention to update this analysis when a future study that meets these benchmarks. Product cost for Apligraf, Dermagraft, Grafix and EpiFix were obtained from the July 2020 CMS ASP Pricing File for Payment Allowance Limits for Medicare Part B Drugs. Product cost for ActiGraft was obtained from RedDress and increased 6\% to be comparable with the other product cost data, since CMS reimburses separately-payable drugs and biologics in the physician office setting at ASP plus 6\%, where ASP is available, and at Wholesale Acquisition Cost (WAC) plus 6\% where ASP is not available.

Other data points required for the analysis are:

i. Skin substitute assignments to High-Cost and LowCost groups were sourced from CMS January 2020 Update of the Hospital Outpatient Prospective Payment System (OPPS). ii. Bundled Hospital Outpatient payments were sourced from CMS July 2020 Addendum B - Final OPPS Payment by HCPCS Code for CY 2020.

iii. Physician Facility and Physician Office fees were sourced from 2020 CMS Physician Fee Schedule.

iv. A standard wound size of 5 square centimeters was used to determine the smallest available product size that would cover the wound.

For Dermagraft and Grafix, the number of product applications was not explicitly stated in the source studies. For Dermagraft, we used 7.1 applications, consistent with the Samsell study. For Grafix we used 6.0 based on the 42 -day median time to heal (Table 2 ). The cost to treat an unhealed DFU of $\$ 14,297$ is an average based on a study citing incremental annual healthcare costs for DFU patients versus non-DFU patients ranging from $\$ 11,710$ for Medicare to $\$ 16,883$ for private insurance cost [18].

Table 2: For Dermagraft and Grafix, the number of product applications was not explicitly stated in the source studies. For Dermagraft, we used 7.1 applications, consistent with the Samsell study. For Grafix we used 6.0 based on the 42-day median time to heal.

\begin{tabular}{|c|c|c|}
\hline (A) & 12-Week Product Cost & Number of Applications over a 12-Week Course of Treatment x Cost per Application \\
\hline (B) & 4-Week Product Cost & $\begin{array}{c}\text { [Lesser of (i) } 4 \text { and (ii) Number of Applications over a 12-Week Course of Treatment] x Cost } \\
\text { per Application }\end{array}$ \\
\hline$(\mathrm{C})$ & $\begin{array}{l}\text { Weighted Average Treatment Cost for Healed } \\
\text { and Unhealed Populations }\end{array}$ & (Percentage Healed x 12-Week Product Cost) + (Percentage Unhealed x \$14,297) \\
\hline (D) & $\begin{array}{l}\text { Medicare Reimbursement Cost, Hospital } \\
\text { Outpatient }\end{array}$ & $\begin{array}{c}\text { (Number of Applications over a 12-Week Course of Treatment x Bundled Hospital Outpa- } \\
\text { tient Payment) + Physician Facility Fee }\end{array}$ \\
\hline$(\mathrm{E})$ & $\begin{array}{l}\text { Medicare Reimbursement Cost, Physician } \\
\text { Office }\end{array}$ & $\begin{array}{c}\text { (Number of Applications over a 12-Week Course of Treatment x Product Cost) + Physician } \\
\text { office Fee }\end{array}$ \\
\hline$(\mathrm{F})$ & $\begin{array}{l}\text { Standardized Effectiveness Analysis, Hospital } \\
\text { Outpatient }\end{array}$ & (Percentage Healed x 100) / (Medicare Reimbursement Cost in HOP Setting/1000) \\
\hline$(G)$ & $\begin{array}{l}\text { Standardized Effectiveness Analysis, Physician } \\
\text { Office }\end{array}$ & $\begin{array}{c}\text { (Percentage Healed x 100) / (Medicare Reimbursement Cost in Physician Office Set- } \\
\text { ting/1000) }\end{array}$ \\
\hline
\end{tabular}

\section{Results}

\section{2-Week Product Cost}

As shown in Table 3 and Chart 1, the 12-week product cost ranges from $\$ 2,120$ to $\$ 7,647$. ActiGraft has a product cost advantage ranging from $\$ 1,245$ to $\$ 5,527$ over 12 weeks. The cost premium for other skin substitutes ranges from 59 to $261 \%$.

\section{Table 3}

\begin{tabular}{|c|c|c|c|c|}
\hline & Red Dress & Organogenesis & Mi Medx & Smith \& Nephew \\
\cline { 2 - 5 } & Acti Graft & Apli graf & Epifix & Grafix core \\
\hline Size & $6 \mathrm{~cm}$ dia. disc & $7 \mathrm{~cm}$ dia. disc & $2 \times 3 \mathrm{~cm}$ & $5 \times 7.5 \mathrm{~cm}$ \\
\hline Square centimeters & 28.3 & 44.2 & 6.0 & 37.5 \\
\hline Number of applications & 8.0 & 2.5 & 3.5 & 6.3 \\
\hline Cost per square cm & $\$ 9.37$ & $\$ 30.47$ & $\$ 160.50$ & $\$ 32.27$ \\
\hline Cost per application & $\$ 265$ & $\$ 1,346$ & $\$ 963$ & $\$ 1,210$ \\
\hline 12 -Week product cost & $\$ 2,120$ & $\$ 3,365$ & $\$ 3,370$ & $\$ 7,647$ \\
\hline Cost premium v Acti Graft & $\$-$ & $59 \%$ & $59 \%$ & $\$ 5,527$ \\
\hline Cost Premium $\%$ & $0 \%$ & 51,250 & $261 \%$ \\
\hline
\end{tabular}


Annals of Reviews and Research

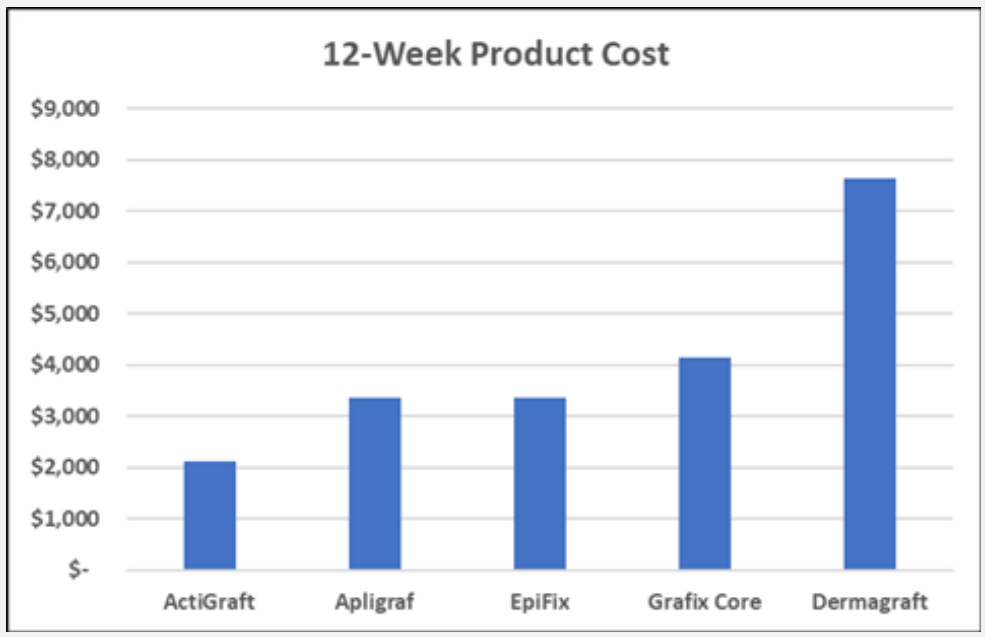

Chart 1

\section{4-Week Product Cost}

4-week product cost is analyzed because studies have shown the 4-week percent area reduction of a wound is a significant predictor of week-12 complete healing [19]. As shown in Table
4 and Chart 2, the 4-week product cost ranges from $\$ 1,060$ to $\$ 4,840$. ActiGraft has a product cost advantage ranging from $\$ 1,710$ to $\$ 3,780$ over 4 weeks. The cost premium for other skin substitutes ranges from 161 to $357 \%$.

Table 4

\begin{tabular}{|c|c|c|c|c|c|}
\hline & Red Dress & Organogenesis & Mi Medx & Smith \& Nephew & Organogenesis \\
\cline { 2 - 6 } & Acti Graft & Apli graf & Epifix & Grafix core & Dermagraft \\
\hline Cost per application & $\$ 265$ & $\$ 1,346$ & $\$ 963$ & $\$ 693$ & $\$ 1,210$ \\
\hline Treatment/4weeks & 4.0 & 2.5 & 3.5 & 4.0 & 4.0 \\
\hline Cost per 4 weeks & $\$ 1,060$ & $\$ 3,365$ & $\$ 2,370$ & $\$ 2,770$ & $\$ 4,840$ \\
\hline Cost premium v Acti Graft & $\$-$ & $\$ 2,305$ & 218 & $\$ 1,710$ & $\$ 3,780$ \\
\hline Cost Premium $\%$ & $0 \%$ & $217 \%$ & $161 \%$ & $357 \%$ \\
\hline
\end{tabular}

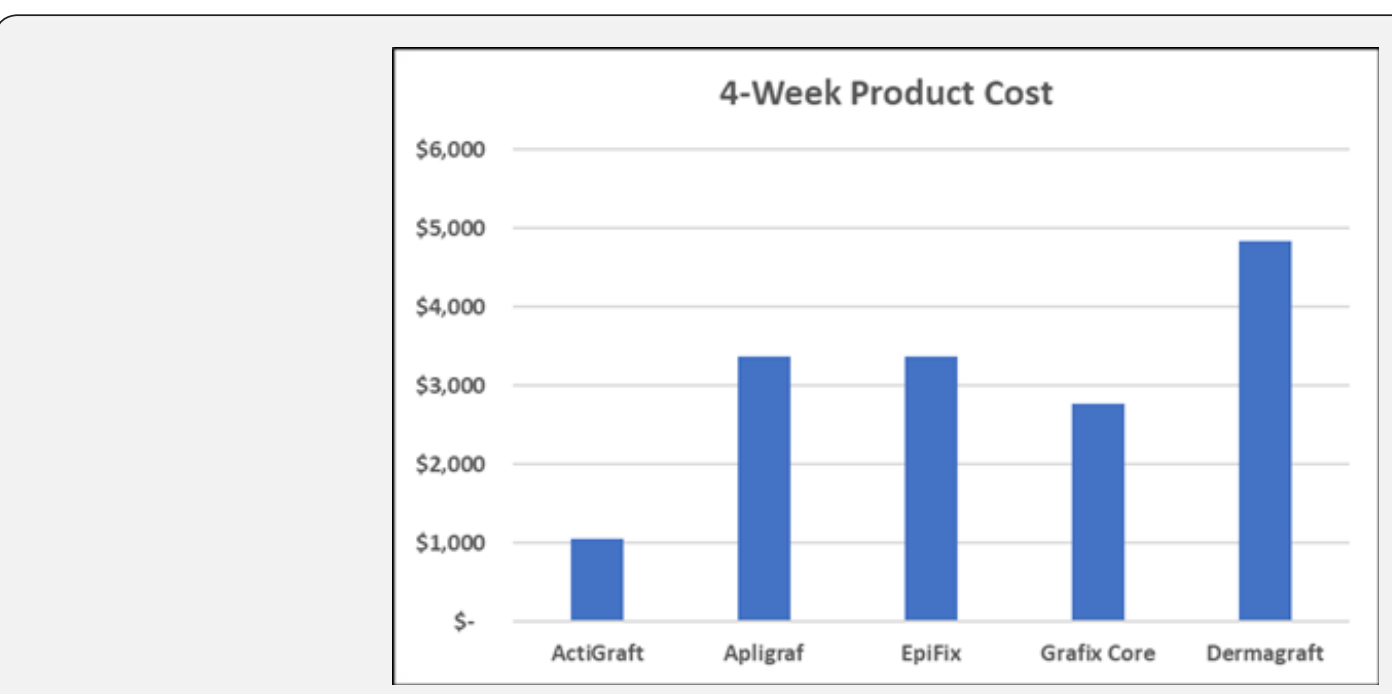

Chart 2 


\section{Weighted Average Treatment Cost for Healed and Unhealed Populations}

This weighted average cost is analyzed to account for both healed and unhealed patient populations, with \$14,297 assigned to the incremental annual cost to treat a DFU patient versus a non DFU patient. This calculation is understated in that the cost to treat the healed population is a product cost only, excluding other treatment costs such as physician and staff labor. As shown in Table 5 and Chart 3 , the WAVG treatment cost ranged from $\$ 5,505$ to $\$ 11,783$. ActiGraft has a cost advantage ranging from $\$ 2,504$ to $\$ 6,278$. The cost premium for other skin substitutes ranges from 45 to $114 \%$

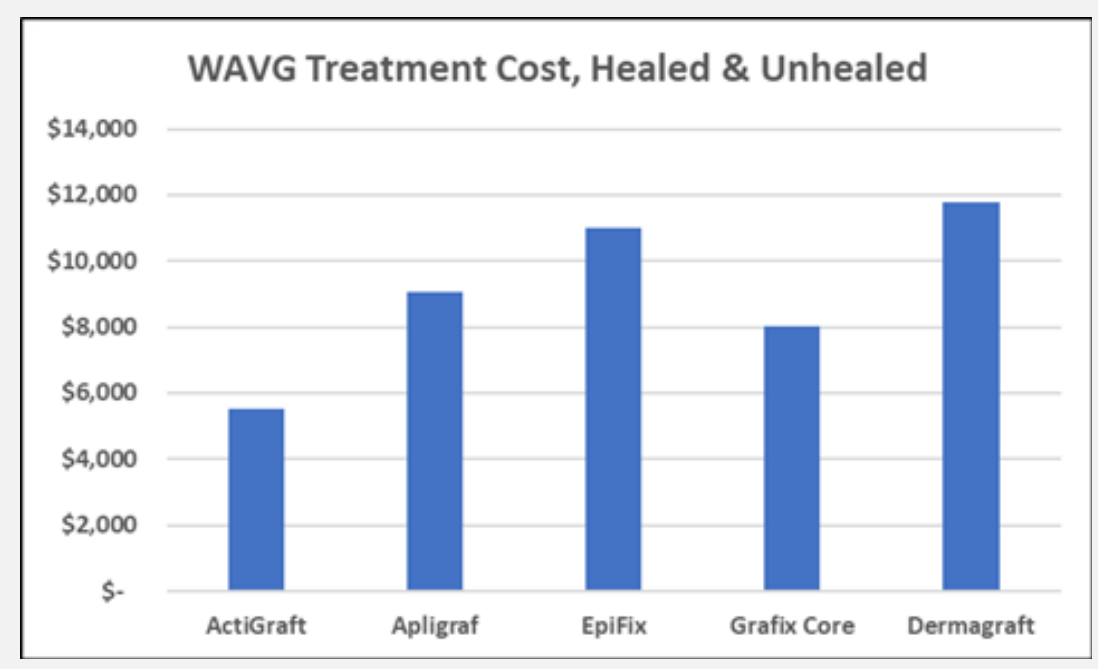

Chart 3

Table 5

\begin{tabular}{|c|c|c|c|c|c|}
\hline & Red Dress & Organogenesis & Mi Medx & Smith \& Nephew & Organogenesis \\
\hline & Acti Graft & Apli graf & Epifix & Grafix core & Dermagraft \\
\hline \multicolumn{6}{|l|}{ 12-week efficacy } \\
\hline \%healed & $72 \%$ & $48 \%$ & $30 \%$ & $62 \%$ & $38 \%$ \\
\hline \%unhealed & $28 \%$ & $52 \%$ & $70 \%$ & $38 \%$ & $62 \%$ \\
\hline \multicolumn{6}{|l|}{ Treatment cost } \\
\hline 12 week procduct cost & $\$ 2,210$ & $\$ 3,365$ & $\$ 3,370$ & $\$ 4,155$ & $\$ 7,647$ \\
\hline Population unhealed & $\$ 14,297$ & $\$ 14,297$ & $\$ 14,297$ & $\$ 14,297$ & $\$ 14,297$ \\
\hline WAVG cost of treatment & $\$ 5,505$ & $\$ 9,049$ & $\$ 11,019$ & $\$ 8,009$ & $\$ 11,783$ \\
\hline Cost premium v Acti Graft & $\$-$ & $\$ 3,544$ & $\$ 5,514$ & $\$ 2,504$ & $\$ 6,278$ \\
\hline Cost Premium \% & $0 \%$ & $64 \%$ & $100 \%$ & $45 \%$ & $114 \%$ \\
\hline
\end{tabular}

Medicare Reimbursement Cost - Hospital Outpatient and Physician Office Settings

Medicare reimbursement cost is analyzed to estimate the relative cost of different products to the Medicare system. As shown in Table 6 and Chart 4, the Medicare Reimbursement Cost ranges from $\$ 4,065$ to $\$ 10,343$ in the hospital outpatient setting, and from $\$ 2,275$ to $\$ 7,802$ in the physician office setting. ActiGraft has a cost advantage ranging from $\$ 81$ to $\$ 6,279$ in the hospital outpatient setting and from $\$ 1,245$ to $\$ 5,527$ in the physician office setting. The cost premium for other skin substitutes ranges from 2 to $154 \%$ in the hospital outpatient setting and from 55 to
$243 \%$ in the physician office setting.

\section{Standardized Effectiveness Analysis}

The Standardized Effectiveness Analysis (SEA) is another metric to assess the relationship between product efficacy and cost. It calculates the number of DFUs healed out of 100 patients per $\$ 1,000$ of Medicare Reimbursement Cost. As shown in Table 7 and Chart 5, SEA ranges from 4 to 18 patients per 100 healed in the Hospital Outpatient Setting, and 5 to 32 patients per 100 healed in the Physician Office setting. ActiGraft has the greatest SEA in both settings. 
Annals of Reviews and Research

Table 6

\begin{tabular}{|c|c|c|c|c|c|}
\hline & Red Dress & Organogenesis & Mi Medx & Smith \& Nephew & Organogenesis \\
\hline & Acti Graft & Apli graf & Epifix & Grafix core & Dermagraft \\
\hline \multicolumn{6}{|l|}{ Hospital Output patient } \\
\hline High/Low cost assignment & Low & High & High & High & High \\
\hline Number of applications & 8.0 & 2.5 & 3.5 & 6.0 & 6.3 \\
\hline X Bundled HOP payment & $\$ 497$ & $\$ 1,623$ & $\$ 1,623$ & $\$ 1,623$ & $\$ 1,623$ \\
\hline Subtotal & $\$ 3,976$ & $\$ 4,057$ & $\$ 5,680$ & $\$ 9,736$ & $\$ 10,255$ \\
\hline Plus physician facility fee & $\$ 88$ & $\$ 88$ & $\$ 88$ & $\$ 88$ & $\$ 88$ \\
\hline Total cost & $\$ 4,065$ & $\$ 4,145$ & $\$ 5,768$ & $\$ 9,825$ & $\$ 10,343$ \\
\hline Cost premium v Acti Graft & $\$-$ & $\$ 81$ & $\$ 1,703$ & $\$ 5,760$ & $\$ 6,279$ \\
\hline Cost premium \% & $0 \%$ & $2 \%$ & $42 \%$ & $142 \%$ & $154 \%$ \\
\hline \multicolumn{6}{|l|}{ Physician Office } \\
\hline Number of applications & 8.0 & 2.5 & 3.5 & 6.0 & 6.3 \\
\hline X Cost per application & $\$ 265$ & $\$ 1,346$ & $\$ 963$ & $\$ 693$ & $\$ 1,120$ \\
\hline Subtotal & $\$ 2,120$ & $\$ 3,365$ & $\$ 3,370$ & $\$ 4,155$ & $\$ 7,647$ \\
\hline Plus physician office fee & $\$ 155$ & $\$ 155$ & $\$ 155$ & $\$ 155$ & $\$ 155$ \\
\hline Total cost & $\$ 2,275$ & $\$ 3,520$ & $\$ 3,525$ & $\$ 4,310$ & $\$ 7,802$ \\
\hline Cost premium v Acti Graft & $\$-$ & $\$ 1,245$ & $\$ 1,250$ & $\$ 2,035$ & $\$ 5,527$ \\
\hline Cost premium \% & $0 \%$ & $55 \%$ & $55 \%$ & $89 \%$ & $243 \%$ \\
\hline
\end{tabular}

Table 7

\begin{tabular}{|c|c|c|c|c|c|}
\hline & Red Dress & Organogenesis & Mi Medx & Smith \& Nephew & Organogenesis \\
\cline { 2 - 6 } & Acti Graft & Apli graf & Epifix & Grafix core & Dermagraft \\
\hline \%healed & $72 \%$ & $48 \%$ & $30 \%$ & $62 \%$ & $38 \%$ \\
\hline Hospital outpatient cost & 4,065 & 4,145 & 5,768 & 9,825 & 4 \\
\hline \#healed per 100 per \$1,000 & 18 & 12 & 5 & 6 & 4,343 \\
\hline Physician 0ffice cost & 2,275 & 3,520 & 3,525 & 4,310 & 7,802 \\
\hline \#healed per 100 per \$1,000 & 32 & 14 & 9 & 14 & 5 \\
\hline
\end{tabular}

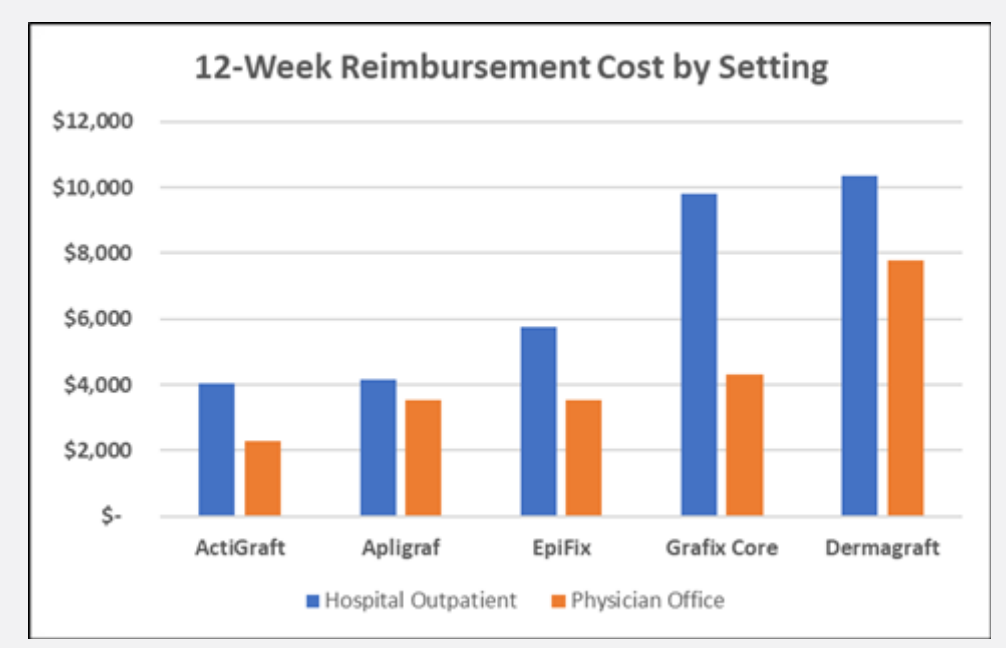

Chart 4 


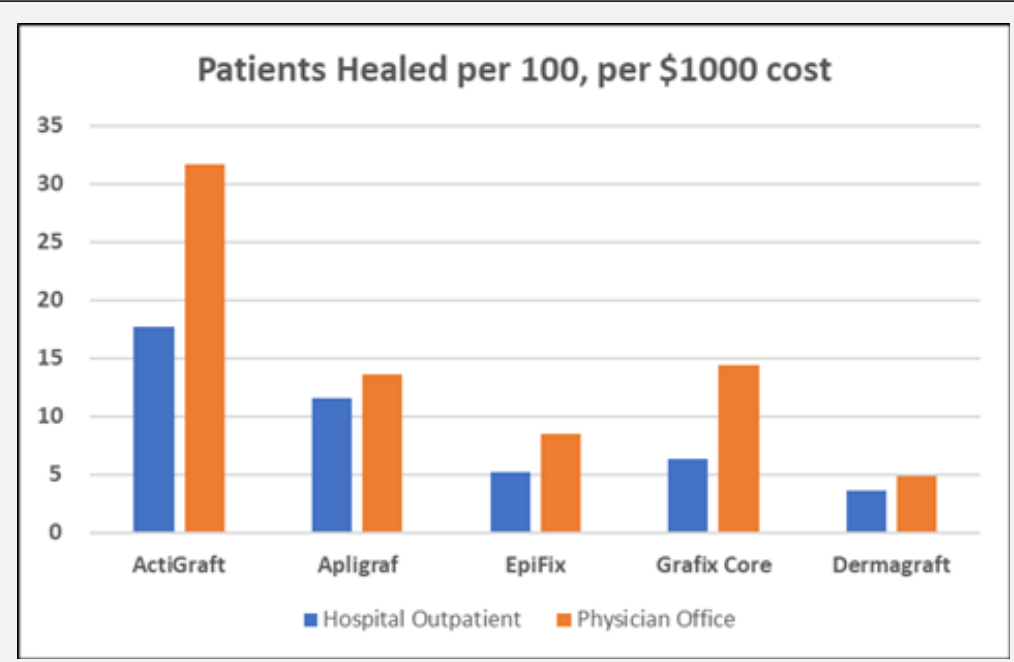

Chart 5

Scatter Plot - 12-Week Product Cost versus Complete Healing Efficacy

Chart 6 illustrates the relationship between 12 -week product cost and complete healing efficacy. ActiGraft offers the lowest cost while delivering the highest healing efficacy Chart 6.

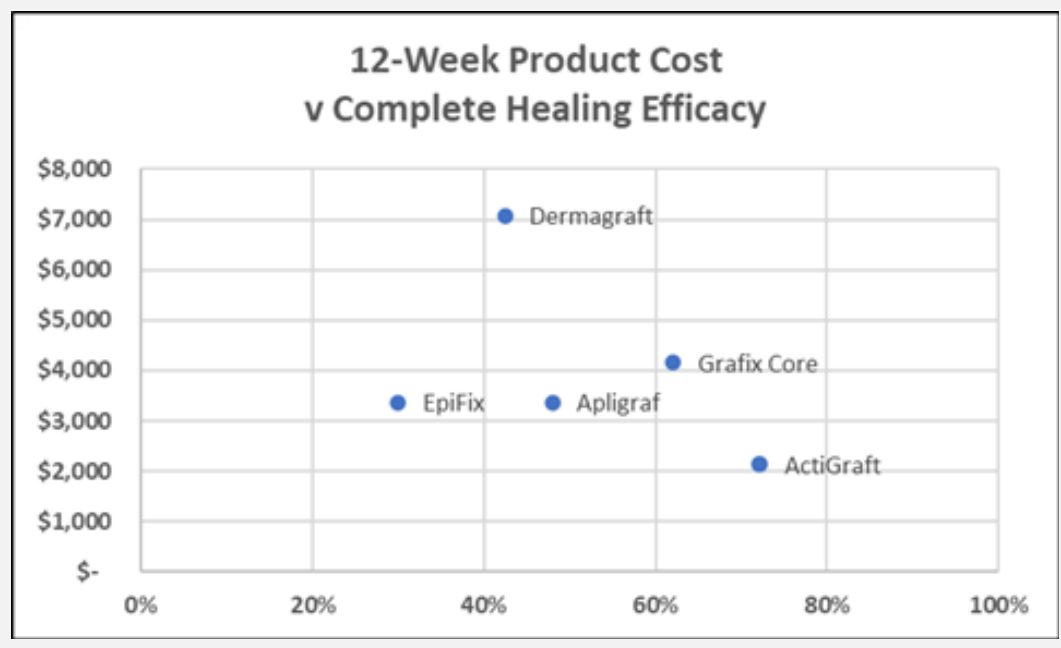

Chart 6

Scatter Plot - Cost per Square Centimeter versus Complete Healing Efficacy

Chart 7 illustrates the relationship between Cost per $\mathrm{cm}^{2}$ and complete healing efficacy. ActiGraft offers the lowest cost per $\mathrm{cm}^{2}$ while delivering the highest healing efficacy Chart 7.

\section{Other Considerations}

\section{Storage}

The following table summarizes storage considerations for each product. ActiGraft and Epifix may be stored at room temperature, while Apligraf, Dermagraft and Grafix have special climate requirements (Table 8).

\section{Inventory}

The following table summarizes product size availability. ActiGraft, Apligraf and Dermagraft are available in one size, simplifying inventory management. EpiFix and Grafix are available in multiple sizes, which is advantageous for minimizing product wastage, but requires maintaining a relatively larger product inventory at the point-of-care (Table 9). 


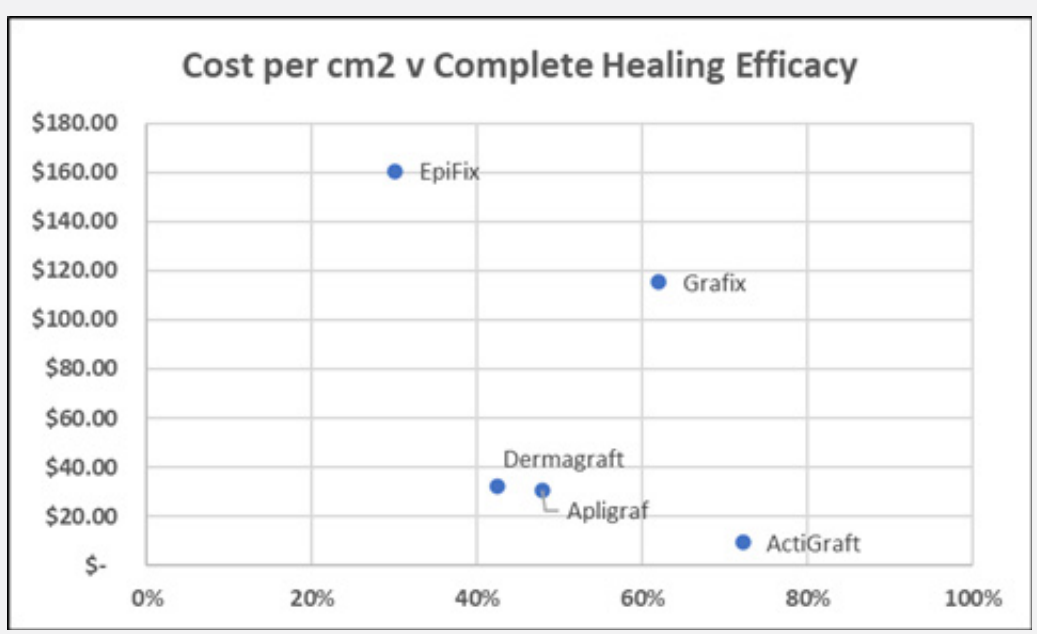

\section{Chart 7}

Table 8

\begin{tabular}{|c|c|}
\hline Product & Storage \\
\hline ActiGraft & Room temperature \\
\hline Apligraf & Must be maintained at $68-73^{\circ} \mathrm{F}$ \\
\hline EpiFix & Room temperature \\
\hline Grafix & Must remain frozen at $-112^{\circ} \mathrm{F}$ \\
\hline Dermagraft & Must remain frozen at $-103 \pm 18^{\circ} \mathrm{F}$ \\
\hline
\end{tabular}

Table 9

\begin{tabular}{|c|c|}
\hline Product & Sizes \\
\hline ActiGraft & $6 \mathrm{~cm}$ diameter disc \\
\hline Apligraf & \begin{tabular}{c}
$7.5 \mathrm{~cm}$ diameter disc \\
\hline EpiFix
\end{tabular} \\
\hline $\begin{array}{c}14 \mathrm{~mm} \text { disk } / 16 \mathrm{~mm} \text { disk } / 2 \times 3 \mathrm{~cm} / 4 \times 4 \mathrm{~cm} / 5 \times 6 \\
\mathrm{~cm} 42\end{array}$ \\
\hline Grafix & $\begin{array}{c}16 \mathrm{~mm} \text { disk } / 1.5 \times 2 \mathrm{~cm} / 2 \times 3 \mathrm{~cm} / 3 \times 4 \mathrm{~cm} / 5 \times 5 \\
\mathrm{~cm} 43\end{array}$ \\
\hline Dermagraft & $5 \times 7.5 \mathrm{~cm}$ \\
\hline
\end{tabular}

\section{Wastage}

ActiGraft is autologous, prepared from the patient's own whole blood at the point of care. As a result, there is no biologic material wastage. Other skin substitutes generate wastage or must be maintained in inventory in a variety of sizes so that biologic material is used economically. For example, Apligraf is available solely in a $44.2 \mathrm{~cm}^{2}$ disc, which would result in $39.2 \mathrm{~cm}^{2}$ wastage for a wound lesser than $5 \mathrm{~cm}^{2}$; while EpiFix must be carried in inventory in a variety of sizes (5 sheet/membrane sizes and 3 particulate sizes in order to minimize wastage at the point of care [20-30].

\section{Conclusion}

This head-to-head comparison showed that ActiGraft was more cost efficient as an advanced therapy for DFU's when compared to the 4 other advanced products investigated. A 12week product cost of the CPT's analyzed ranged from $\$ 2,120$ to $\$ 7,647$. ActiGraft had a product cost advantage ranging from $\$ 1,245$ to $\$ 5,527$ over 12 weeks. The cost premium for other skin substitutes ranged from 59 to $261 \%$. The 4 -week product cost ranged from $\$ 1,060$ to $\$ 4,840$. ActiGraft had a product cost advantage ranging from $\$ 1,710$ to $\$ 3,780$ over 4 weeks. The cost premium for other skin substitutes ranged from 161 to $357 \%$. The weighted average treatment cost (WAVG) ranged from $\$ 5,505$ to $\$ 11,783$. ActiGraft had a cost advantage ranging from $\$ 2,504$ to $\$ 6,278$. The cost premium for other skin substitutes ranged from 45 to $114 \%$. ActiGraft had a cost advantage ranging from $\$ 81$ to $\$ 5,760$ in the hospital outpatient setting and from $\$ 1,245$ to $\$ 4,959$ in the physician office setting. The cost premium for other skin substitutes ranged from 2 to $142 \%$ in the hospital outpatient setting and from 55 to $218 \%$ in the physician office setting. Standardized Effective Analysis (SEA) ranged from 4 to 18 patients per 100 healed in the hospital outpatient setting, and 5 to 32 patients per 100 healed in the physician office setting. ActiGraft had the greatest SEA in both settings. ActiGraft offered the lowest cost per $\mathrm{cm}^{2}$ while delivering the highest healing efficacy. Unlike other CTP's in this analysis, ActiGraft is autologous, prepared from the patient's own whole blood at the point of care and therefore has no biologic material wastage. It should be noted that the ActiGraft data sourced from a pilot study presents limitations for comparison with studies meeting Samsell's criteria. It is the authors' intention to update this analysis when additional published studies meet this benchmark [31-40].

\section{References}

1. Zelen C (2013) An evaluation of dehydrated human amniotic membrane allografts in patients with DFUs. Journal of Wound Care 22(7): 347-351.

2. Geiss LS, Li Y, Hora I, Albright A, Rolka D, et al. (2018) Resurgence of diabetes-related nontraumatic lower extremity amputation in the young and middle-aged adult US population. 
3. (2011) Centers for Disease Control and Prevention, National Center for Chronic Disease and Health Promotion. National Diabetes Fact Sheet: National estimates and general information on diabetes and prediabetes in the United States, Atlanta, GA: Centers for Disease Control \& Prevention.

4. Brownrigg JRW, Apelqvist J, Bakker K, Schaper NC, Hinchliffe RJ, et al. (2013) Evidence-based managed of PAD \& the Diabetic Foot. Euro J Vasc and Endovascular Surg 45(6): 673-681.

5. Samsell B, McLean J, Cazzell S (2018) Health economics for treatment of diabetic foot ulcers: a cost-effectiveness analysis of eight skin substitutes. Journal of Wound Care North American Supplement 28(9).

6. Accessed on August $4^{\text {th }}, 2020$ Rau, Jordan. New Round of Medicare Readmission Penalties Hits 2,583 Hospitals. Kaiser Health News.

7. Margolis DJ, Kantor J, Berlin JA (1999) Healing of diabetic neuropathic foot ulcers receiving standard treatment. A meta-analysis. Diabetes Care 22(5): 692-695.

8. Cheryl S, Knispel J, Lopes M (2012) Clinical and cost efficacy of advanced wound matrices for venous ulcers. J Manag Care Pharm 18(5): 375-384

9. Sen C, Gordillo G, Roy S, Kirsner R, Lambert L (2009) Human skin wounds: A major and snowballing threat to public health and the economy. Wound Repair and Regeneration 17(6): 763-771.

10. Tavis MJ, Thornton J, Danet R, Bartlett RH (1978) Current status of skin substitutes. Surg Clin North Am 58(6): 1233-1248.

11. Snyder RJ, Kasper MA, Patel K (2018) Safety and Efficacy of an Autologous Blood Clot Product in the Management of Texas $1 \mathrm{~A}$ or $2 \mathrm{~A}$ Neuropathic Diabetic Foot Ulcers: A Prospective, Multicenter, Open Label Pilot Study. Wounds 30(7): 84-89.

12. Edmonds M (2009) European and Australian Apligraf Diabetic Foot Ulcer Study Group. Apligraf in the treatment of neuropathic diabetic foot ulcers. Int J Low Extrem Wounds 8(1): 11-18.

13. Falanga V, Margolis D, Alvarez O, Auletta M, Maggiacomo F, et al. (1998) Rapid healing of venous ulcers and lack of clinical rejection with an allogeneic cultured human skin equivalent. Human Skin Equivalent Investigators Group. J Arch Dermatol 134(3): 293-300.

14. Marston WA, Hanft J, Norwood P, Pollak R (2003) Dermagraft Diabetic Foot Ulcer Study Group. The efficacy and safety of Dermagraft in improving the healing of chronic diabetic foot ulcers: results of a prospective randomized trial. Diabetes Care 26(6): 1701-1705.

15. Snyder D, Sullivan N, Margolis D (2020) Skin Substitutes for Treating Chronic Wounds [Internet]. Rockville (MD): Agency for Healthcare Research and Quality (US). Table D-2, Acellular/Dermal replacement from human placental membrane.

16. Zelen CM, Serena TE, Gould L (2016) Treatment of chronic diabetic lower extremity ulcers with advanced therapies: a prospective, randomised, controlled, multi-centre comparative study examining clinical efficacy and cost. Int Wound J 13: 272-2782.

17. Lavery LA, Fulmer J, Shebetka KA (2014) The efficacy and safety of Grafix $(\circledR)$ for the treatment of chronic diabetic foot ulcers: results of a multi-centre, controlled, randomised, blinded, clinical trial. International Wound Journal 11(5): 554-560.

18. Rice JB, Desai U, Cummings AKG (2014) Burden of Diabetic foot Ulcers for Medicare and Private Insurers. Diabetes Care p. 37(1): 651-658.

19. Sheehan P, Jones P, Caselli A (2003) Percent Change in Wound Area of Diabetic Foot Ulcers Over a 4-Week Period Is a Robust Predictor of Complete Healing in a 12-Week Prospective Trial. Diabetes Care 26: 1879-1882.
20. Narres, M, Kvitkina, T, Claessen, H (2017) Incidence of lower extremity amputations in the diabetic compared with the non-diabetic population: A systemic review PLoS One 12(8): e0182081

21. (2014) National Diabetes Statistics Report: Estimates of Diabetes and Its Burden in the United States. Centers for Disease Control and Prevention.

22. Lee C, Rogers (2008) The Right to Bear Legs-An Amendment to Healthcare: How Preventing Amputations Can Save Billions for the US Health-care System. Journal of the American Podiatric Medical Association 98(2): 166-168.

23. Nussbaum SR, Carter MJ, Fife CE, et al. (2018) An Economic Evaluation of the Impact, Cost, and Medicare Policy Implications of Chronic Nonhealing Wounds. Value Health 21(1): 27-32.

24. Carter MJ (2010) Cost-effectiveness research in wound care: Definitions, approaches, and limitations. Wound Management \& Prevention 56(11).

25. Panzer RJ, Gitomer RS, Greene WH, Webster PR, Landry KR, et al. (2013) Increasing demands for quality measurement. JAMA 310: 1971-1980.

26. Snyder, Robert J DPM (2016) Fife, Caroline MD; Moore, Zena PhD Components and Quality Measures of DIME (Devitalized Tissue, Infection/Inflammation, Moisture Balance, and Edge Preparation) in Wound Care, Advances in Skin \& Wound Care 29(5): 205-215.

27. Klevens RM, Edwards JR, Richards CL, Horan TC, Gaynes RP, et al. (2007) Estimating health care-associated infections and deaths in U.S. hospitals, 2002. Public health reports Washington DC 1974 122(2): 160-166.

28. Rau, Jordan (2020) Preeminent Hospitals Penalized Over Rates of Patients' Injuries. Kaiser Health News.

29. Kazmi B, Inglefield CJ, Lewis MP (2009) Autologous cell therapy: current treatments and future prospects. Wounds 21(9): 234-242.

30. Snyder RJ (2005) Treatment of nonhealing ulcers with allografts. Clin Dermatol 23(4): 388-395.

31. Curran MP, Plosker GL (2002) Bilayered bioengineered skin substitute (Apligraf): A review of its use in the treatment of venous leg ulcers and diabetic foot ulcers. Bio Drugs 16(6): 439-455.

32. Fetterolf DE, Snyder RJ (2012) Scientific and clinical support for the use of dehydrated amniotic membrane in wound management. Wounds 24(10): 299-307.

33. Eming S A, Martin, P, Tomic-Canic M (2014) Wound repair and regeneration: mechanisms, signaling, and translation. Science translational medicine 6(265): $265 \mathrm{sr} 6$.

34. Zelen C (2013) An evaluation of dehydrated human amniotic membrane allografts in patients with DFUs. Journal of Wound Care 22(7): 347-351

35. Raspovic KM, Wuklich DK, Naiman DQ, Lavery LA, Kirsner RS, et al. (2018) Effectiveness of viable cryopreserved placental membranes for management of diabetic foot ulcers in a real-world setting. Wound Repair Regeneration 26: 213-220.

36. Kirsner RS, Sabolinski ML, Parsons NB (2015) Comparative effectiveness of a bioengineered living cellular construct vs. a dehydrated human amniotic membrane allograft for the treatment of diabetic foot ulcers in a real-world setting. Wound Rep Reg 23(5): 737-744.

37. Marston WA, Pollak R, Hanft J,Norwood P (2003) The Efficacy and Safety of Dermagraft in Improving the Healing of Chronic Diabetic Foot Ulcers. Diabetes Care 26(6): 1701-1705. 
38. Kraus, I, Sabolinski ML, Skornicki M, Parsons NB (2017) The Comparative Effectiveness of a Human Fibroblast Dermal Substitute versus a Dehydrated Human Amnion/Chorion Membrane Allograft for the Treatment of Diabetic Foot Ulcers in a Real-world Setting. Wounds 29(5): 125-132.
39. https://www.petepetit.com/pete-petit-professional-blog/2015/ mimedx-products-epifix.html

40. http://www.osiris.com/grafix/healthcare-professionals/

Your next submission with Juniper Publishers will reach you the below assets

- Quality Editorial service

- Swift Peer Review

- Reprints availability

- E-prints Service

- Manuscript Podcast for convenient understanding

- Global attainment for your research

- Manuscript accessibility in different formats

( Pdf, E-pub, Full Text, Audio)

- Unceasing customer service

Track the below URL for one-step submission https://juniperpublishers.com/online-submission.php 